\title{
La genèse des études du glacier de Sarennes et le premier quart de siècle 1949-1974
}

\author{
The beginning of the Sarennes Glacier studies and the first \\ century quarter 1949-1974 \\ par L. de Crécy
}

The observations in the Sarennes Glacier started in 1948/49, after some front recognitions at the beginning of the century. Five measurement points were chosen at diverse altitudes. Corrrelations between glacial balances and climate data were established but nothing is totaly definite.

Le Jubilé est à la mode ! Mais, d'après le Lévitique (ch $25,8)$, l'année qui suit "sept semaines d'années soit sept fois sept ans", c'est-à-dire l'année sacrée du jubilé, il est dit "qu'on ne sème ni ne récolte". Les observations glaciologiques de Sarennes ayant commencé sous leur forme actuelle lors de la saison 1948/1949, c'est la saison 1998/1999 qui aurait pu être tenue comme l'année sacrée du jubilé, celle où l'on n'aurait pas dû récolter la moindre observation ; c'est donc avec plaisir et soulagement que je constate que malgré le précepte biblique, il y a bien eu des observations l'an dernier !

Mais j'arrête là mon commentaire du Lévitique, puisque c'est de la genèse qu'on m'a demandé de vous entretenir aujourd'hui, la genèse des observations sur le glacier de Sarennes s'entend !

\section{I - LES OBSERVATIONS ANCIENNES}

L'intérêt pour les glaciers est né au milieu du XIX $\mathrm{X}^{\mathrm{e}}$ siècle chez les géologues alpins constatant que des blocs rocheux isolés avaient parcouru des distances parfois considérables depuis le site dont ils étaient manifestement originaires. On s'avisa que la glace était susceptible de déformation plastique et, en climat tempéré, d'un véritable écoulement par fusion-regel à la base. On eut vite fait de retrouver dans l'environnement du massif alpin les traces évidentes des principaux épisodes glaciaires du Quaternaire.

Il était intéressant, dès lors, d'observer ces mouvements en détail à la période actuelle. Au cours des deux dernières décennies du siècle, on s'attacha d'abord à fixer l'état des lieux par une description détaillée des appareils glaciaires subsistant dans la chaîne alpine ; en France, P. Guillemain et le Prince Roland Bonaparte furent les artisans de cet inventaire.
Ils donnent en particulier une carte d'ensemble du massif des Grandes Rousses datant de 1891. Dès cette époque, le glacier de Sarennes est estimé en léger recul mais les repères placés sur le front en septembre 1891 ne seront pas retrouvés.

A Sarennes, l'état des lieux ne fut établi avec précision que par le levé de 1905/1906 conduit par des universitaires grenoblois, Flusin, Jacob et Offner sur l'ensemble du massif des Grandes Rousses : en 1906 Sarennes mesure 109 ha 8 et son front a reculé de $6 \mathrm{~m}$ entre août 1905 et août 1906 .

\section{II — LES FORESTIERS ET LES GLACIERS}

Les mouvements glaciaires sont lents : pour les mettre en évidence, il faut les observer d'année en année avec des méthodes simples et invariables mais aussi une exceptionnelle et fastidieuse continuité qui relèvent plus des consignes permanentes d'une administration que de chercheurs imaginatifs.

Or l'administration des Eaux et Forêts devait justement être bientôt conduite à s'intéresser de près aux études de glaciologie : le 11 juillet 1892 , la rupture d'une poche d'eau sous-glaciaire au petit glacier de Tête Rousse dans le massif du Mont Blanc provoque la destruction du village de SaintGervais faisant 192 morts. Le jeune service de Restauration des Terrains en Montagne était spécialisé dans la lutte contre l'érosion torrentielle classique. Mais quand un torrent se trouve subitement dans l'obligation d'évacuer en quelques heures $200000 \mathrm{~m}^{3}$ d'eau bientôt chargées de $800000 \mathrm{~m}^{3}$ de matériaux solides, les dégâts dépassent évidemment toute prévision.

C'est à la suite de cet accident et de quelques autres menaces d'origine glaciaire que les forestiers ont entrepris la surveillance des langues des principaux glaciers alpins : 
mesures des avances et reculs des fronts glaciaires, levés estivaux systématiques d'un certain nombre de profils, permettant d'observer gonflements et affaissements

A Sarennes, où n'existe pratiquement pas de langue terminale, les services forestiers se contentent de quelques reconnaissances du front : le point le plus bas était en 1905 à l'altitude de $2776,2 \mathrm{~m}$. Il était remonté à $2790,2 \mathrm{~m}$ en 1911 , mais redescendu à $2789,2 \mathrm{~m}$ en 1923 et même à $2788,7 \mathrm{~m}$ en 1927, pour remonter fortement, nous le verrons, à $2826,4 \mathrm{~m}$ en 1949

\section{III — DES OBSERVATIONS DU TYPE SCANDINAVE}

C'est en effet à cette année 1949 que remontent les observations systématiques dont nous célébrons le "jubilé".

Le Conservateur des Eaux et Forêts Jean Cherrey est passionné de glaciologie. Il vient de créer, avec l'Inspecteur Général Jean Messines du Sourbier, ce qui n'est encore que la "sous-section de glaciologie" à l'intérieur de la Société Hydrotechnique de France .

Il constate que les mesures traditionnelles de langues glaciaires sont mal adaptées à un glacier de cirque de type pyrénéen ; en revanche, le glacier de Sarennes présente une relativement faible dénivelée $(360 \mathrm{~m})$ entre les points le plus haut et le plus bas, il est peu encaissé entre des falaises peu élevées mais raides parce que constituées de solides schistes cristallins granulitisés perchés en haut d'un anticlinal hercynien.

La glace occupe les $2 / 3$ du bassin d'alimentation. L'exposition est uniforme vers le Sud. La dynamique de la glace y est manifestement très faible, difficilement capable de remplacer l'excès d'ablation estivale à l'aval de la ligne des névés par un éventuel surplus d'accumulation à l'amont, quand ce surplus existe.

Ces caractéristiques le rapprochent plus, sur bien des points, des glaciers scandinaves que des glaciers alpins de vallée. Il était naturel que M. Cherrey fût tenté d'y implanter les méthodes des glaciologues scandinaves mises au point par Ahlman et son école au cours des années 1930.

Le principe est de mesurer en différents points du glacier, chaque point étant supposé représentatif d'une tranche d'altitude, d'une part, l'accumulation nouvelle en fin de saison hivernale au dessus du niveau de l'année précédente, puis, d'autre part, les étapes de l'ablation par des visites successives en saison chaude en suivant la consommation d'abord de la neige de l'hiver, puis, au moins aux stations aval, celle d'une certaine épaisseur de glace.

En chaque point on établit ainsi un bilan local "accumulation moins ablation". Sur un glacier supposé en équilibre, Ahlman établit qu'à la "ligne de névés" l'accumulation est égale à l'ablation, la somme de ces deux grandeurs constituant le régime du glacier.

\section{IV $\square$ LE CHOIX DE SARENNES}

Ces mesures supposent donc, avec un matériel parfois encombrant, de pouvoir assurer une visite en fin mai, puis de 5 à 8 visites entre juin et fin septembre quelles que soient les circonstances météorologiques. C'eût été peut-être un pari risqué en bien des sites de haute montagne mais c'était aisé à Sarennes à partir de l'Alpe d'Huez par les chalets de la mine de l'Herpie (ce le sera bientôt encore plus, voir trop. dans quelques années après la création du téléphérique du Pic du Lac Blanc).

En 1949, des agents d'EdF (Schmidt, Laronde) avaient, par ailleurs, réalisé un levé précis du glacier qui donnera une excellente base de départ aux nouvelles observations : la surface est de 82 ha dans un bassin versant mesurant au total 132 ha, soit une perte de 27 ha en surface (un peu plus du quart) par rapport à la surface du glacier de 1906 (qui était de 109,2 ha). Dès le printemps 1949, Cherrey a inauguré les mesures de l'accumulation de la saison hivernale 1948/1949 puis le suivi de l'ablation selon une méthode qui ne variera pratiquement plus.

\section{V — LE DÉROULEMENT DES OBSERVA- TIONS ANNUELLES}

Répartition des points de mesure : 5 points de mesures on été établis, censés représenter chacun le comportement d'une certaine tranche d'altitude et donc une certaine surface du glacier :

En 1949 :

- la station 1 était à $2890 \mathrm{~m}$ d'altitude représentant environ $5 \%$ de la surface du glacier :

- la station 2 était à 2934 m et représentait 10\% de la surface glaciaire :

- la station 3, à $2970 \mathrm{~m}$ représentait $25 \%$ de la surface du glacier :

- les stations 4 et 5 , à 3005 et 3056 m d'altitude représentaient chacune $30 \%$ de la surface du glacier.

La localisation topographique de ces stations restera inchangée, même si leurs altitudes viennent à diminuer au cours des années, du fait de l'affaissement continu du glacier

La première mesure a lieu d'ordinaire fin mai - début juin ; c'est celle qui va, en général, fixer la valeur de l'accumulation de la saison : la neige recouvre tout, on prélève verticalement avec une sonde cylindrique une colonne de neige que l'on pèse. Rapporté à la surface de la section de la sonde, ce poids représente la valeur en eau de la couverture de neige, valeur qu'on a l'habitude d'exprimer en centimètres. La difficulté est de s'assurer que l'on a bien sondé jusqu'au niveau de la glace ou de la vieille neige telle qu'il était en fin de la saison d'ablation de l'été précédent. A cet effet, si l'on n'a pas retrouvé de jalon datant de la saison précédente, on réalise à la pelle une tranchée verticale devant le point du sondage.

Les mesures de la marche de l'ablation s'échelonnent ensuite toutes les deux à trois semaines jusqu'à fin septembre. Elles comportent d'abord, exactement comme cidessus, la mesure de la valeur en eau de la couverture de neige subsistant au-dessus de la glace ancienne. L'ablation de la neige se mesure par différence avec les observations précédentes. Mais dès que possible, et en tout cas s'il subsiste moins d'un mètre de neige, on implante un jalon métallique enfoncé d'environ deux mètres dans la glace et l'on repère avec précision le dépassement du jalon au-dessus de la glace : une simple lecture, au prochain passage donnera le montant de l'ablation de glace entre deux passages .

Ces mesures traditionnelles sont finalement extrêmement simples, mais elles exigent le transport d'un équipement minimum en sonde, balance romaine, jalons et, bien sûr, 
pelles et barre foreuse. Une partie de ce matériel est dans la cabane construite au front du glacier par M. Cherrey, mais comment ne pas évoquer le guide Marius Sarret de l'Alpe d'Huez, qui, trente ans durant, conduira les reconnaissances avec le responsable du moment et deviendra finalement le meilleur garant de l'homogénéité des mesures.

Après les deux premières campagnes $(1948 / 1949$ et 1949/1950) la relève fut prise par Louis Garavel avec ce qui était la $7^{\text {ème }}$ section de recherche forestière. Les innombrables changements d'appellations que devait subir cette section avant d'aboutir au sein de l'actuel CEMAGREF ne l'ont pas empêché de garder fidèlement et d'appliquer avec rigueur les quelques consignes simples d'observation de Sarennes. D'où la remarquable continuité qui ont fait leur valeur, plus de 50 ans durant. Chaque année, la réunion de la section de glaciologie de la SHF comprenait le compte rendu classique sur les observations de Sarennes que les auditeurs avaient souvent la possibilité de comparer avec les observations plus élaborées du laboratoire grenoblois de glaciologie du CNRS réalisaient sur le glacier symétrique de Saint Sorlin, de l'autre côté du massif des Grandes Rousses .

\section{VI - LES CORRÉLATIONS BILANS GLACIAIRES ET CLIMAT}

Tout naturellement, les observations sur Sarennes conduisaient leurs auteurs à se référer aux données climatiques locales. Cherrey avait déjà souligné le contraste entre les deux premières saisons d'observations dont la première avait été marquée par un déficit exceptionnel de l'accumulation (59 $\mathrm{cm}$ de valeur en eau au printemps 1949 pour $168 \mathrm{~cm}$ au printemps 1950).

Dès 1955 , Garavel comparait, dans la revue forestière $\left(\mathrm{n}^{\circ} 1\right.$ de 1955) les 5 premiers bilans de Sarennes aux données climatiques grenobloises en utilisant les indices trimestriels d'aridité (tels que définis par de Martonne) d'automne, d'hiver et de printemps pour rendre compte de l'accumulation et le même indice d'été pour l'ablation .

Les premiers résultats étaient prometteurs, mais, sur une plus longue période, on risquait des déconvenues car le mois de juin qui est inclus dans l'indice d'aridité de printemps, intervenait comme facteur d'accumulation alors que, certaines années, il participe manifestement à l'ablation de la saison chaude.

D'où ma tentative en 1963 de caractériser l'accumulation par les précipitations à Grenoble des mois d'octobre à mai et l'ablation par le rapport entre la température moyenne des 4 mois d'été (juin à septembre) et la précipitation totale annuelle. Appliquée aux 14 premiers cycles de Sarennes, la méthode donnait des résultats encourageants mais qui ne furent pas parfaitement confirmés par certains cycles ultérieurs.

\section{VII — LE RÔLE PARTICULIER DES PRÉCIPITATIONS DE JUIN?}

Ce sera en faisant intervenir tout spécialement et avec un fort coefficient la pluviosité du mois de juin que Serge Martin obtiendra la meilleure corrélation du climat de la plaine (Lyon Bron) avec les bilans de Sarennes des 26 premiers cycles (1948/1949 à 1974/75).

A la réflexion, le rôle particulier joué à Sarennes par les précipitations de juin souligne le fait que - à la différence des glaciers scandinaves étudiés par Ahlman - la principale source d'énergie provoquant la fusion y est, sous un ciel toujours dégagé et à exposition sud, bien plus le rayonnement solaire que la température de masses d'air peu chargées d'humidité : en cas de fortes précipitations en juin (toujours sous forme solide à $3000 \mathrm{~m}$ ), la neige fraîche possède un albédo de 0,9 et renvoie $90 \%$ de l'énergie reçue, protégeant efficacement la majeure partie du glacier contre la fusion durant tout le mois de juillet, parfois jusqu'en août. Au contraire, après un mois de juin peu arrosé, la vieille neige puis la glace n'ont plus qu'un albédo de l'ordre de $50 \%$ lorsque arrivent les mois chauds de juillet et d'août ; d'où une fusion beaucoup plus rapide.

Mais je m'avance peut-être beaucoup! Une nouvelle tentative de corrélation entre bilans glaciaires et facteurs météorologiques devrait prendre en compte non plus 5 , ni 14 , ni 26 bilans annuels, mais 50 ! 